\title{
A composição corporal está associada com o risco de quedas e medo de cair em pacientes em hemodiálise
}

\section{Body composition is associated with the risk of falls and fear of falling in hemodialysis patients}

\author{
Heitor S. Ribeiro' (1) \\ Helton L. Carvalho 2 (1) \\ Gustavo Ítalo Dourado ${ }^{3}$ (1) \\ Lucas Almeida 4 \\ Marvery P. Duarte ${ }^{5}$ \\ André Bonadias Gadelha ${ }^{6}$ (i) \\ Antônio Inda-Filho ${ }^{7}$ \\ Aparecido P. Ferreira ${ }^{8}$ (1)
}

\footnotetext{
${ }^{1}$ Autor para correspondência. Universidade de Brasília (Brasília). Distrito Federal, Brazil. heitorsiqueira95@gmail.com 2,7,8 Centro Universitário ICESP (Brasília). Distrito Federal, Brazil. h.carvalho9595@gmail.com, indafilho@hotmail.com, cidopimentel@gmail.com 3-5Universidade de Brasília (Brasília). Distrito Federal, Brazil.gussttavoitallo@gmail.com, lucasalmeidaedf@gmail.com, marveryp@gmail.com ${ }^{6}$ Instituto Federal de Educação, Ciência e Tecnologia Goiano (Urutaí). Goiás, Brasil. andrebonadias@gmail.com
}

RESUMO | INTRODUçÃO: Pacientes com doença renal crônica (DRC) submetidos à hemodiálise (HD) apresentam alterações musculoesqueléticas e de composição corporal que podem levar à redução do equilíbrio, velocidade de caminhada e capacidade de realizar as atividades de vida diária, aumentando o risco de quedas. OBJETIVO: Investigar a associação da composição corporal com o risco de quedas e medo de cair em pacientes com DRC submetidos à HD. MATERIAIS E MÉTODOS: Estudo transversal com amostra de 40 pacientes em HD. Para o medo de cair e risco de quedas foram aplicados a Escala Internacional de Eficácia de Quedas e o QuickScreen Clinical Falls Risk Assessment. A amostra foi estratificada no percentil 50 para comparação da composição corporal de acordo com o risco de quedas e medo de cair. Para comparação entre os grupos, empregou-se o teste t de Student independente, e a correlação de Spearman para associar o risco de quedas e medo de cair com a composição corporal. O nível de significância adotado foi de $p<0,05$. RESULTADOS: Correlação moderada positiva entre a gordura corporal com o medo de cair $(r=0,47)$ e risco de quedas $(r=0,42)$ e correlação moderada negativa entre a massa magra com o medo de cair ( $r=-$ $0,51)$ e risco de quedas $(r=-0,45)$. O grupo com maior risco de quedas apresentou maior gordura corporal $(36,8 \pm 8,2$ vs $30,9 \pm 6,9 ; p=0,043)$. 0 grupo com maior medo de cair obteve menor massa magra $(41,6 \pm 9,2$ vs 52,0 7,$6 ; p=0,004)$. CONCLUSÃo: As variáveis de composição corporal associaram-se ao risco de quedas e ao medo de cair em pacientes com DRC submetidos à HD.

PALAVRAS-CHAVE: Acidentes por quedas. Composição corporal. Força muscular. Adiposidade. Insuficiência renal crônica.
ABSTRACT | INTRODUCTION: Patients with chronic kidney disease (CKD) on hemodialysis (HD) experience musculoskeletal and body composition changes that may lead to reduced balance, gait speed and ability to perform activities of daily living, thus increasing the risk of falls. OBJECTIVE: To investigate the association between body composition with the risk of falls and fear of falling in HD patients. METHODS: Crosssectional study of 40 patients on HD. Fear of falling and risk of falls were assessed with the International Efficacy Scale of Falls and the QuickScreen Clinical Falls Risk Assessment. The sample was stratified at the 50th percentile to compare body composition according to the risk of falls and fear of falling. For comparison between groups, the independent Student $t$ test was used and Spearman's correlation to associate the risk of falls and fear of falling with body composition. The level of significance was $p<0.05$ RESULTS: Moderate positive correlation between body fat with fear of falling ( $r=0.47)$ and risk of falling $(r=0.42)$, and moderate negative correlation between lean mass with fear of falling $(r=-0.51)$ and risk of falls $(r=-0.45)$. The group with the highest risk of falls had higher body fat $(36.8 \pm 8.2$ vs $30.9 \pm 6.9 ; p=0.043)$. The group with the highest fear of falling had less lean mass (41.6 \pm 9.2 vs $52.0 \pm 7.6$; $p=0.004)$. CONCLUSION: Body composition is associated with the risk of falls and the fear of falling in patients with CKD on HD.

KEYWORDS: Accidental falls. Body composition. Muscle strength. Adiposity. Chronic renal insufficiency. 


\section{Introdução}

Pacientes com doença renal crônica (DRC) submetidos à hemodiálise (HD) apresentam alterações musculoesqueléticas e de composição corporal que podem levar à redução da capacidade de realizar as atividades de vida diária (AVD). A diminuição da força e da massa muscular, decorrentes da DRC, resultam em redução do equilíbrio, velocidade de caminhada e aumento do risco de quedas 1, .

Na população idosa geral, quedas e resultados adversos associados, são um problema de saúde substancial associado a um risco significativo de morte ${ }^{3}$. Em pacientes com DRC em HD, as quedas também tem se tornado cada vez mais prevalentes e preocupantes, seja em pacientes idosos ou não ${ }^{4-7}$. Os fatores de risco associados ao risco de quedas aumentado em pacientes com DRC incluem a alta prevalência de comorbidades, múltiplas terapias medicamentosas, instabilidade hemodinâmica e metabólica induzida pela frequência do tratamento e fragilidade ${ }^{8,9}$.

Muito se sabe a respeito dos aspectos funcionais, tais como força muscular, função física e equilíbrio postural, e a sua associação com o maior risco de quedas e medo de cair em diversas populações especiais, incluindo a DRC ${ }^{10-13}$. Todavia, não está claro na literatura qual o papel das variáveis de composição corporal nos mecanismos fisiológicos da ocorrência de quedas, e as poucas evidências existentes em idosos apresentam resultados conflituosos ${ }^{14}$.

Da mesma forma, até o presente momento não há evidências que elucidem a relação da composição corporal e quedas em pacientes com DRC em HD. Sendo assim, o presente estudo teve como objetivo investigar a associação da composição corporal com o risco de quedas e medo de cair em pacientes com DRC submetidos à HD.

\section{Métodos}

Trata-se de um estudo transversal, realizado em uma clínica particular de HD, na cidade de Brasília DF, entre os meses de janeiro e março de 2018. Este estudo foi aprovado pelo Comitê de Ética em Pesquisa da Universidade Católica de Brasília, número 2.497.191/2018 (CAAE 79677617.5.0000.0029).

\section{População do estudo}

Os participantes foram recrutados através de convites feitos pelos pesquisadores e pelo corpo médico da clínica por meio de conversas durante as sessões de HD. Foram elegíveis para o estudo aqueles que estivessem realizando tratamento hemodialítico por tempo superior a três meses, liberação da equipe médica, possuir 18 anos de idade ou mais e assinar o termo de consentimento livre esclarecido (TCLE). Foram excluídos os voluntários que apresentavam doença cardiopulmonar grave (por exemplo, infarto do miocárdio recente ou angina instável), ter faltado a última sessão de diálise antes do início das avaliações físicas e restrição musculoesquelético que impedissem a realização dos testes. A população elegível foi composta por 55 pacientes, destes, 40 aceitaram participar do estudo (figura 1).

Figura 1. Fluxograma do estudo

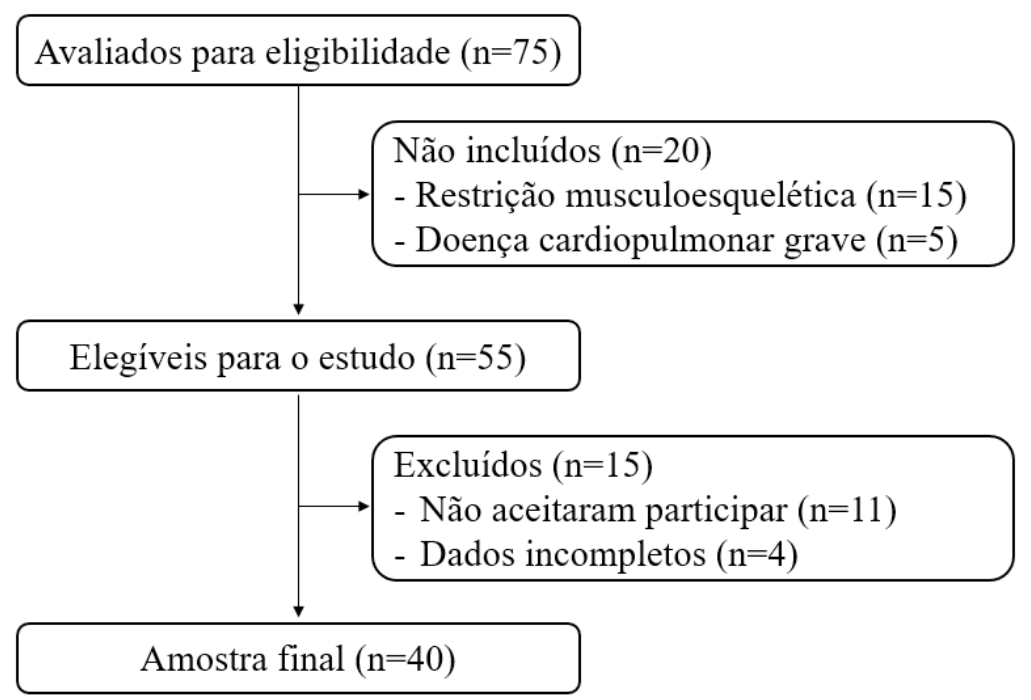




\section{Procedimentos gerais}

Os procedimentos de avaliação foram realizados em duas visitas à clínica. Na primeira visita, foi explicado a todos os pacientes de forma detalhada os objetivos, procedimentos, benefícios e possíveis riscos do estudo. Logo após, assinaram o TCLE e responderam ao questionário sociodemográfico. Uma semana após, na segunda visita, os participantes incluídos realizaram as seguintes avaliações, divididas em três momentos: I) avaliações antropométricas e de composição corporal; II) força de preensão palmar (FPP) e; III) Escala Internacional de Eficácia de Quedas (FES-I) e QuickScreen Clinical Falls Risk Assessment para avaliar o medo de cair e risco de quedas, respectivamente ${ }^{15,16}$. Todas as avaliações foram feitas ao final da sessão de HD por um avaliador previamente treinado em sala climatizada.

\section{Estratificação dos grupos}

A estratificação dos grupos deu-se pelo percentil 50 superior e inferior através dos resultados obtidos pela FES-I e QuickScreen. O resultado do percentil 50 superior para ambas as avaliações foi considerado como os grupos com maiores medo de cair e risco de quedas. Por outro lado, o resultado do percentil 50 inferior foi considerado como os grupos com menores medo de cair e risco de quedas, respectivamente.

\section{Avaliações antropométricas}

Inicialmente, a massa corporal foi mensurada por meio de uma balança digital portátil (FilizolaTM, Beyond Technology, PL - 200, São Paulo, Brasil), com resolução de 0,1 quilograma (kg). Neste procedimento, foi solicitado aos participantes a retirar qualquer acessório que pudesse influenciar na medida (exemplo: anel, brincos, bonés, relógio e outros), pés descalços e o peso distribuído igualmente nas duas pernas, ao final, o peso foi registrado em $\mathrm{kg}$. Em seguida, a estatura foi medida por meio de um estadiômetro de parede com resolução de 1 centímetro (FilizolaTM, Beyond Technology, PL - 200, São Paulo, Brasil). Para esta medida, foi empregada a posição anatômica, mantendo-se pés unidos, calcanhares, nádegas e troncos encostados na parede, palmas das mãos voltadas para frente e a cabeça posicionada ao plano de Frankfurt. Através das medidas de massa corporal e estatura, foi possível realizar o cálculo do índice de massa corporal (IMC) por meio da seguinte fórmula: IMC = peso/altura*altura. Todas as avalições antropométricas foram realizadas em uma sala climatizada e separada da sala de HD, respeitando as recomendações impostas pelos fabricantes e por um avaliador experiente.

\section{Composição corporal}

A composição corporal foi medida por meio da bioimpedância tetrapolar (Byodinamics, ®310e, São Paulo, Brasil). Para executar o procedimento, foi solicitado aos pacientes que ficassem na posição em decúbito dorsal, assim, foi possível a colocação dos eletrodos nas partes distais dos pés e mãos direitos. Para os pacientes que possuíssem marca-passo, a realização do procedimento não foi realizada, respeitando as recomendações do fabricante. As medidas da massa magra (kg), massa gorda (kg), gordura corporal (\%) e taxa metabólica basal (TMB) foram obtidas através da avaliação.

\section{Força de preensão palmar}

Para avaliação da FPP, utilizou-se o equipamento dinamômetro hidráulico analógico, calibrado previamente as avaliações (Jamar, J00105, Illinois, USA). Os participantes foram posicionados sentados, seguindo o preconizado pela Sociedade Americana de Terapeutas de Mão, na qual os quadris e os joelhos se encontram fletidos a $90^{\circ}$, ombro aduzido em posição neutra, cotovelo fletido a $90^{\circ}$ e antebraço em semi-pronação. A pegada no dinamômetro foi ajustada individualmente de acordo com o tamanho das mãos dos voluntários, de forma que a alça ficasse ajustada para que o polegar ficasse ao lado da alça e os demais dedos "abraçando" toda a alça, confortavelmente. A mensuração foi feita no braço não fistulado ou dominante para aqueles com acesso via cateter. Três tentativas foram realizadas, sendo solicitado que 0 indivíduo aplicasse a maior força possível durante 4 segundos para cada uma das tentativas. Foi considerada para análise a medida de maior valor entre as tentativas. Durante a realização do teste, os participantes receberam encorajamento verbal. Para cada tentativa, foi dado um intervalo de 30 segundos. Os resultados foram registrados em $\mathrm{kg} / \mathrm{fl}^{17}$.

\section{Escala Internacional de Eficácia de Quedas}

Para avaliação do risco de quedas, foi utilizada a FES-I. A queda foi definida como ato não intencional de cair no chão ou em nível inferior ao seu plano. A FES-I é um questionário que contém 16 domínios com diferentes atividades de vida diária com quatro possibilidades de respostas e respectivos escores de 1 a 4 ("Nem um pouco preocupado" a "Extremamente 
preocupado"). O escore total pode variar de 16 a 64 (ausência de preocupação a preocupação extrema) em relação às quedas, durante a realização das atividades específicas do questionário ${ }^{15}$.

\section{Quickscreen Clinical Falls Risk Assessment}

O QuickScreen Clinical Falls Risk Assessment trata-se de um questionário validado internacionalmente contendo oito itens: ocorrência de quedas no ano anterior, quantidade de medicamentos em uso - excluindo as vitaminas, utilização de psicotrópicos, avaliação da acuidade visual, teste da sensibilidade periférica, avaliação do equilíbrio, do tempo de reação e da força que verificam a presença ou ausência de fatores de riscos que podem levar a queda, revelando valores em porcentagem (\%) que indicam a probabilidade de cair nos próximos 12 meses. Na presença de 0 ou 1 fator, a probabilidade de cair é de $7 \%, 2$ ou 3 fatores $13 \%$, 4 ou 5 fatores $25 \%$ e 6 ou mais fatores têm $49 \%$ de probabilidade de queda ${ }^{16}$.

\section{Análises bioquímicas}

A coleta sanguínea e as análises bioquímicas de ureia, creatinina e albumina foram realizadas por uma empresa particular especializada, como parte da rotina clínica dos pacientes. Os procedimentos foram realizados ao início da HD, no período matutino e vespertino durante o mês de avaliação do paciente.

\section{Análise estatística}

Os dados foram expressos por meio dos valores da média e desvio padrão ( \pm ). O teste de KolmogorovSmirnov foi utilizado para verificar a normalidade dos dados. O teste t de Student independente foi usado para comparar a composição corporal de acordo com os grupos estratificados para as variáveis risco de queda e medo de cair. Adicionalmente, o teste de correlação de Spearman foi adotado para verificar possíveis associações entre as variáveis de composição corporal e FPP no risco de quedas e medo de cair. O nível de significância estabelecido foi de $95 \%$. Todas as análises estatísticas foram realizadas com o programa Statistical Package for the Social Sciences (SPSS), versão 22.0 (SPSS Inc., Chicago, IL, EUA).

\section{Resultados}

A tabela 1 apresenta os resultados referentes as caraterísticas sociodemográficas e clínicas dos participantes do presente estudo.

Tabela 1. Caracterização dos pacientes em hemodiálise $(n=40)$

\begin{tabular}{|c|c|}
\hline Variáveis & Média \pm DP \\
\hline Idade (anos) & $53,6 \pm 16,0$ \\
\hline Peso corporal (kg) & $68,5 \pm 15,1$ \\
\hline Estatura (m) & $1,64 \pm 0,1$ \\
\hline Circunferência de cintura (cm) & $97,5 \pm 13,3$ \\
\hline $\mathrm{IMC}\left(\mathrm{kg} / \mathrm{m}^{2}\right)$ & $30,1 \pm 5,6$ \\
\hline Massa magra (kg) & $45,6 \pm 9,9$ \\
\hline Massa gorda $(\mathrm{kg})$ & $23,8 \pm 8,8$ \\
\hline Gordura corporal (\%) & $33,8 \pm 8$ \\
\hline TMB (kcal) & $1383,4 \pm 305,4$ \\
\hline Tempo de tratamento (meses) & $29,7 \pm 28,7$ \\
\hline Força de preensão palmar (kgf) & $25,4 \pm 10,1$ \\
\hline Medo de cair (FES-I) & $23,6 \pm 10,7$ \\
\hline Ureia pré HD (mg/dL) & $132,7 \pm 30,8$ \\
\hline Ureia pós HD (mg/dL) & $56,1 \pm 21,7$ \\
\hline Creatinina $(\mathrm{mg} / \mathrm{dL})$ & $9,0 \pm 3,4$ \\
\hline Albumina (g/dL) & $4,1 \pm 0,6$ \\
\hline
\end{tabular}

IMC, índice de massa corporal; TMB, taxa metabólica basal; HD, hemodiálise. 
Um total de 40 participantes fizeram parte do estudo. A maioria dos integrantes eram homens $(60 \% ; n=24)$, apresentavam tempo de tratamento de $29,7 \pm 28,7$ meses e idade média de 53,6 $\pm 16,0$ anos.

A figura 2 demonstra o risco de queda futura em 12 meses de acordo com o QuickScreen Clinical Falls Risk Assessment.

Figura 2. Risco de quedas de pacientes em hemodiálise de acordo com o QuickScreen $(n=40)$



Como observado, apenas $20 \%$ apresentam um baixo risco de quedas (7\%), mesmo valor para o maior risco de quedas (49\%). Somados os riscos de 27 e $49 \%$, há um total de $47,5 \%$ da amostra com risco elevado de quedas.

A tabela 2 apresenta os dados da análise de correlação entre as variáveis de composição corporal e FPP com o medo de cair e risco de quedas.

Tabela 2. Correlação entre variáveis de composição corporal e funcional de acordo com o medo de cair e risco de quedas de pacientes em hemodiálise ( $\mathrm{n}=40$ )

\begin{tabular}{lcc}
\hline \multicolumn{1}{c}{ Variáveis } & Medo de cair & Risco de quedas \\
\hline Massa magra $(\mathrm{kg})$ & $-0,551^{*}$ & $-0,450^{*}$ \\
Massa gorda $(\mathrm{kg})$ & 0,093 & 0,124 \\
Gordura corporal (\%) & $0,473^{*}$ & $0,422^{*}$ \\
TMB (kcal) & $-0,553^{*}$ & $-0,446^{*}$ \\
Força de preensão palmar (kgf) & $-0,406^{*}$ & $-0,155$ \\
\hline
\end{tabular}

TMB, taxa metabólica basal; * $\mathrm{p}<0,05$.

Os achados da tabela 2 revelam associações negativas entre massa magra, TMB e FPP com o medo de cair e risco de quedas, entretanto, a FPP apenas foi significativa com o medo de cair. Ademais, a gordura corporal apresentou associações positivas com o medo de cair e risco de quedas.

A figura 3 apresenta uma comparação do percentual de gordura corporal realizada entre os grupos com menor e maior risco de quedas. 
Figura 3. Comparação da gordura corporal de acordo com o risco de quedas de pacientes em hemodiálise $(n=40)$

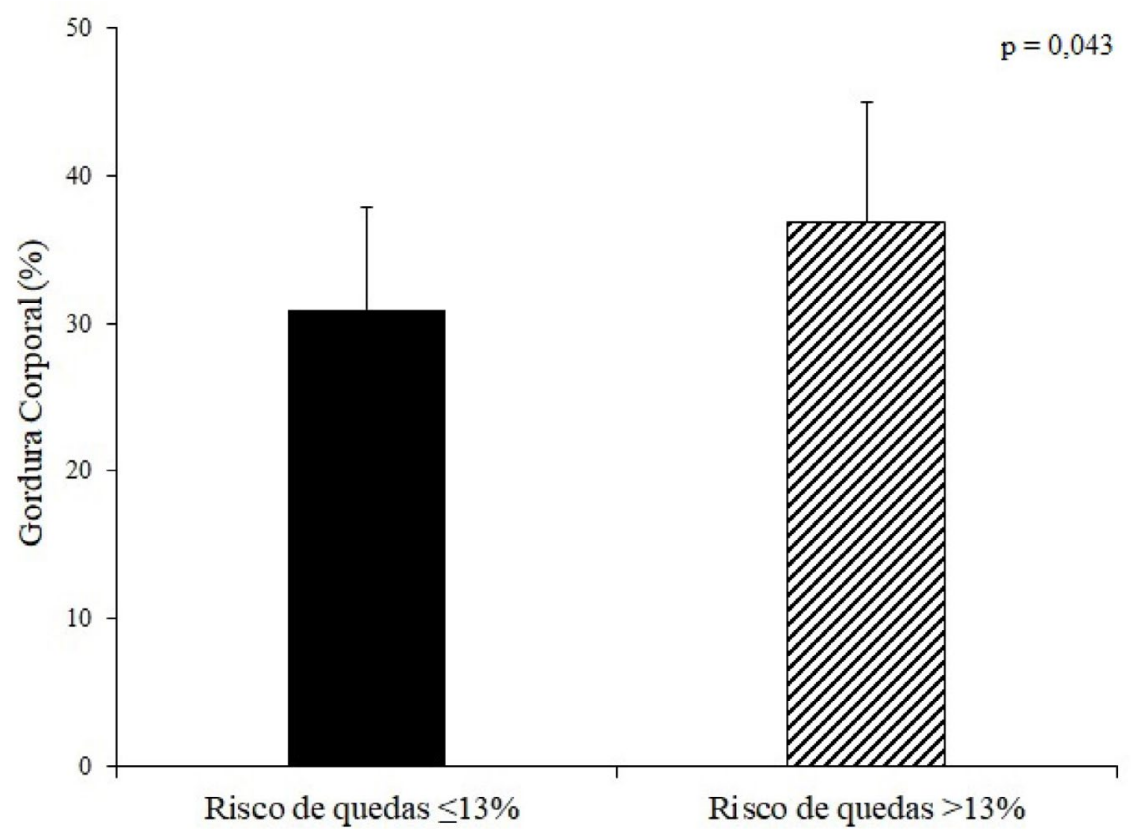

Como visto na figura 3, o grupo com menor risco de quedas apresenta menor gordura corporal quando comparado ao grupo com maior risco de quedas $(36,8 \pm 8,2$ vs $30,9 \pm 6,9 ; p=0,043)$.

A figura 4 mostra uma comparação dos valores de massa magra entre os grupos com menor e maior medo de cair.

Figura 4. Comparação da massa magra de acordo com o medo de cair de pacientes em hemodiálise $(n=40)$

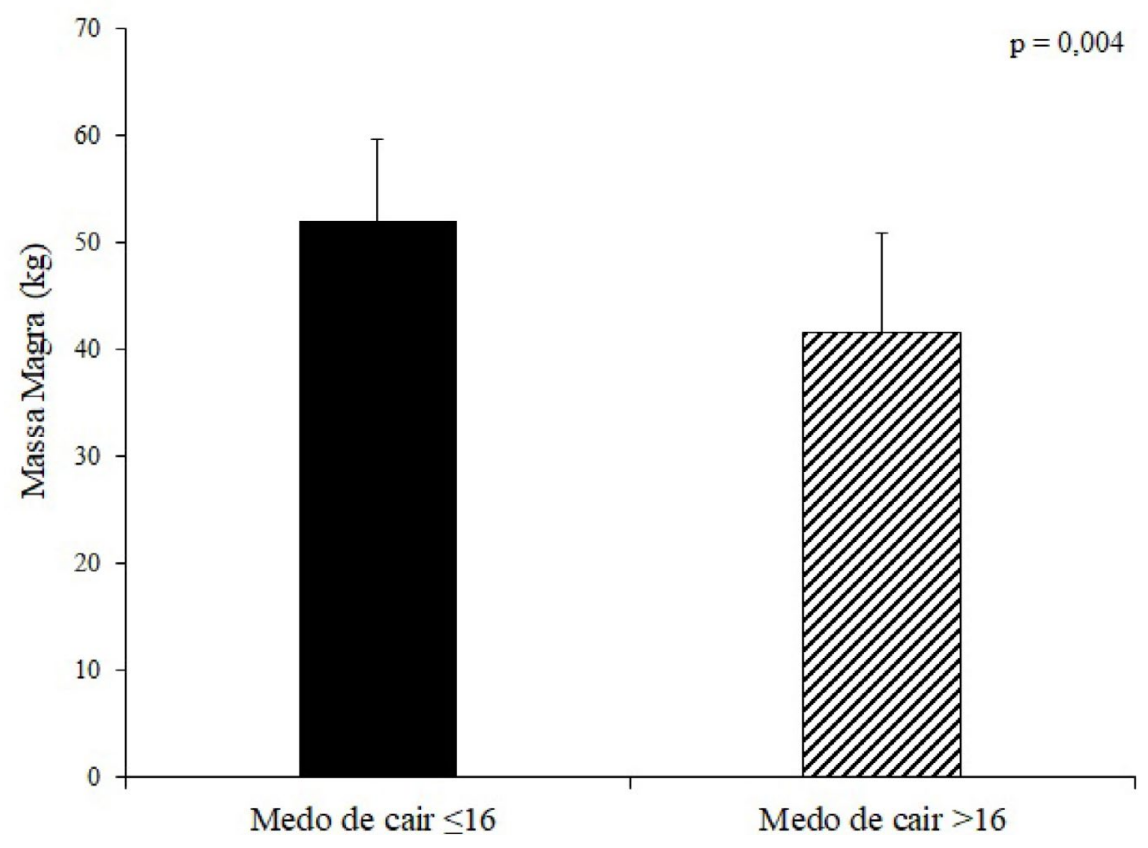

Os resultados apresentados revelam que o grupo com maior medo de cair apresenta menor valor de massa magra em comparação ao grupo com menor medo de cair $(41,6 \pm 9,2$ vs $52 \pm 7,6 ; p=0,004)$. 


\section{Discussão}

Os achados do estudo confirmam a existência da associação entre as variáveis de composição corporal com o medo de cair e risco de quedas em pacientes com DRC em HD. Ao avaliar o risco de quedas, observou-se uma associação moderada à massa magra, gordura corporal e a TMB. Não obstante, o medo cair mostrou-se moderadamente associado à massa magra, gordura corporal, TMB e FPP.

Evidências indicam o risco aumentado de quedas relacionados a pacientes com DRC em HD 2,18. Os achados do presente estudo estão em consonância com a literatura, sendo observado que $47,5 \%$ da amostra encontrou-se com o risco de quedas aumentado. A queda é um dos grandes problemas de morbimortalidade em idosos com DRC, com prevalência de fraturas que variam de 26,9 a $55 \% \frac{19}{}$. Fatores como a idade, sexo, força muscular e comorbidades implicam para o aumento deste risco, ademais, idosos com DRC têm um risco $81 \%$ maior de quedas quando comparados aos que não possuem a doença². Lópes-Soto et al. (2015) apontam um risco aumentado de quedas para pacientes mais velhos em $\mathrm{HD}^{20}$, adicionalmente, os autores destacam que pacientes em HD apresentam maior dependência funcional, o que leva a uma redução da massa e força muscular, consequentemente, aumentando o risco de episódios de quedas 21 .

Grande parte dos estudos que investigam quedas em pacientes com DRC apontam apenas para associações com variáveis funcionais, como a força muscular, equilíbrio e função física, havendo uma lacuna para a compreensão do papel da composição corporal neste fenômeno ${ }^{13,22}$. Em idosos, estudos prévios mostram outras variáveis que se associam e explicam o risco de quedas e medo de cair, tal como a composição corporal, em especial a gordura corporal ${ }^{10,23-25}$. Estas variáveis corroboram com os achados do nosso estudo, sendo observado associações negativas entre a massa magra e TMB com o medo de cair e risco de quedas, e a gordura corporal com associações positivas com o medo de cair e risco de quedas. Além disso, o grupo com menor risco de quedas apresentou menor gordura corporal quando comparado ao grupo com maior risco de quedas, achado semelhante para o grupo com maior medo de cair, o qual apresentou menor valor de massa magra em comparação ao grupo com menor medo de cair.
Como observado, a massa magra esteve mais associada às variáveis de quedas quando comparado a gordura corporal. Estratégias para a manutenção e aumento da massa magra em pacientes com DRC em HD devem ser, portanto, estimuladas a fim de se prevenir possíveis eventos de quedas. Nesse sentido, intervenções com exercício físico, especialmente o treinamento de força, surgem como possíveis modificadores de variáveis da composição corporal associadas ao risco de quedas e medo de cair 21 .

O estudo apresenta limitações pela sua natureza transversal, o que impede inferências de causa-efeito. Assim, são necessárias investigações de acompanhamento para estabelecer relações temporais entre a composição corporal, risco de quedas e medo de cair. O número de voluntários participantes do estudo foi relativamente pequeno, refletindo a dificuldade de acesso à população em HD. Aplicações práticas podem ser compreendidas por meio dos achados do presente estudo, onde medidas simples da composição corporal, de baixo custo, podem ser factualmente aplicadas na rotina clínica para avaliação de pacientes com DRC que, normalmente, apresentam elevado risco de quedas e medo de cair.

\section{Conclusão}

Os resultados observados demonstram que há associação entre as variáveis de composição corporal com o risco de quedas e medo de cair em pacientes com DRC em HD. Diante disso, as avaliações de composição corporal devem ser vistas com atenção por parte dos profissionais de saúde e, adicionalmente, é importante a implementação de intervenções capazes de diminuir o risco de quedas e medo de cair nesta população.

\section{Agradecimentos}

Agradecemos ao Grupo de Estudos em Fisiologia do Exercício e Saúde (GEFES) pelo apoio à coleta de dados, à equipe da Clínica Nefrolntensimed Nefrologia por fornecer tudo à nossa pesquisa e à Coordenação de Aperfeiçoamento de Pessoal de Nível Superior (CAPES) pela bolsa de estudos de mestrado ao Heitor Ribeiro. Este estudo foi financiado pela Fundação de Apoio à Pesquisa do Distrito Federal (FAPDF) (concessão 0193.001.558/2017). 


\section{Contribuições dos autores}

Ribeiro HS participou da concepção, delineamento do método, análise dos dados e redação do artigo. Carvalho HL, Dourado G, Almeida LS e Duarte MP participaram da coleta de dados e redação do artigo. Gadelha AB participou da redação e aprovação final do artigo. Inda-Filho AA e Ferreira AP participaram da concepção, delineamento do método e aprovação do artigo.

\section{Conflitos de interesses}

Nenhum conflito financeiro, legal ou político envolvendo terceiros (governo, empresas e fundações privadas, etc.) foi declarado para nenhum aspecto do trabalho submetido (incluindo, mas não se limitando a subvenções e financiamentos, participação em conselho consultivo, desenho de estudo, preparação de manuscrito, análise estatística, etc.).

\section{Referências}

1. Hiraki K, Yasuda T, Hotta C, Izawa KP, Morio Y, Watanabe S et al. Decreased physical function in pre-dialysis patients with chronic kidney disease. Clin Exp Nephrol. 2013;17(2):225-31. doi: $10.1007 /$ s10157-012-0681-8

2. Kistler BM, Khubchandani J, Jakubowicz G, Wilund K, Sosnoff J. Falls and fall-related injuries among US adults aged 65 or older with chronic kidney disease. Prev Chronic Dis. 2018;15(6):82. doi: 10.5888/pcd15.170518

3. Tinetti ME, Speechley M, Ginter SF. Risk Factors for Falls among Elderly Persons Living in the Community. N Engl J Med. 1988;319(26):1701-7. doi: 10.1056/NEJM198812293192604

4. Cook WL, Tomlinson G, Donaldson M, Markowitz SN, Naglie $\mathrm{G}$, Sobolev $\mathrm{B}$ et al. Falls and fall-related injuries in older dialysis patients. Clin J Am Soc Nephrol. 2006;1(6):1197-204. doi: 10.2215/ C]N.01650506

5. Sutcliffe BK, Bennett PN, Fraser SF, Mohebbi M. The deterioration in physical function of hemodialysis patients. Hemodial Int. 2018;22(2):245-53. doi: 10.1111/hdi.12570

6. Delgado C, Shieh S, Grimes B, Chertow GM, Dalrymple LS, Kaysen GA et al. Association of Self-Reported Frailty with Falls and Fractures among Patients New to Dialysis. Am J Nephrol. 2015;42(2):134-40. doi: 10.1159/000439000

7. Abdel-Rahman EM, Turgut $F$, Turkmen $K$, Balogun RA. Falls in elderly hemodialysis patients. QJM. 2011;104(10):829-38. doi: 10.1093/qjmed/hcr108

8. Papakonstantinopoulou K, Sofianos I. Risk of falls in chronic kidney disease. J Frailty, Sarcopenia Falls. 2017;02(02):33-8. doi: 10.22540/JFSF-02-033
9. Fabbian F, Giorgi AD, Borrego MAR, López-Soto PJ. Frailty, chronic kidney disease and falls: A vicious circle. J Caser Rep Images Med. 2017;3:14-17. doi: 10.5348/Z09-2017-33-ED-5

10. Neri SGR, Harvey LA, Tiedemann A, Gadelha AB, Lima RM. Obesity and falls in older women: Mediating effects of muscle quality, foot loads and postural control. Gait Posture. 2020;77:138-43. doi: 10.1016/j.gaitpost.2020.01.025

11. Gadelha AB, Neri SGR, Nóbrega OT, Pereira JC, Bottaro $M$, Fonsêca $A$ et al. Muscle quality is associated with dynamic balance, fear of falling, and falls in older women. Exp Gerontol. 2018;104:1-6. doi: 10.1016/j.exger.2018.01.003

12. Gadelha AB, Neri SGR, Bottaro M, Lima RM. The relationship between muscle quality and incidence of falls in older communitydwelling women: An 18-month follow-up study. Exp Gerontol. 2018;110:241-6. doi: 10.1016/j.exger.2018.06.018

13. Zanotto T, Mercer TH, Van Der Linden ML, Rush R, Traynor $J P$, Petrie $C J$ et al. The relative importance of frailty, physical and cardiovascular function as exercise-modifiable predictors of falls in haemodialysis patients: A prospective cohort study. BMC Nephrol. 2020;21:99. doi: 10.1186/s12882-020-01759-z

14. Neri SGR, Oliveira JS, Dario AB, Lima RM, Tiedemann A. Does obesity increase the risk and severity of falls in people aged 60 years and older? A systematic review and metaanalysis of observational studies. J Gerontol A Biol Sci Med Sci. 2020;75(5):952-960. doi: 10.1093/gerona/glz272

15. Friedman SM, Munoz B, West SK, Rubin GS, Fried LP. Falls and fear of falling: Which comes first? A longitudinal prediction model suggests strategies for primary and secondary prevention. J Am Geriatr Soc. 2002;50(8):1329-35. doi: 10.1046/j.15325415.2002.50352.x

16. Tiedemann A, Lord SR, Sherrington C. The development and validation of a brief performance-based fall risk assessment tool for use in primary care. J Gerontol A Biol Sci Med Sci. 2010;65(8):896-903. doi: 10.1093/gerona/glq067

17. Delanaye $P$, Quinonez $K$, Buckinx $F$, Krzesinski J-M, Bruyère $\mathrm{O}$. Hand grip strength measurement in haemodialysis patients: before or after the session? Clin Kidney J. 2018;11(4):555-558. doi: 10.1093/ckj/sfx139

18. Nickolas TL, McMahon DJ, Shane E. Relationship between moderate to severe kidney disease and hip fracture in the United States. J Am Soc Nephrol. 2006;17(11):3223-32. doi: 10.1681/ ASN.2005111194

19. Morley J. Frailty: Diagnosis and management. J Nutr Health Aging. 2011;15(8):667-70. doi: 10.1007/s12603-011-0338-4

20. López-Soto PJ, De Giorgi A, Senno E, Tiseo R, Ferraresi A, Canella $C$ et al. Renal disease and accidental falls: A review of published evidence. BMC Nephrol. 2015;16(1). doi: $10.1186 /$ s12882-015-0173-7 
21. Parker K. Intradialytic Exercise is Medicine for Hemodialysis Patients. Curr Sports Med Rep. 2016;15(4):269-75. doi: 10.1249/ JSR.0000000000000280

22. Goto NA, Weststrate ACG, Oosterlaan FM, Verhaar MC, Willems HC, Emmelot-Vonk MH et al. The association between chronic kidney disease, falls, and fractures: a systematic review and meta-analysis. Osteoporos Int. 2020;31(1):13-29. doi: $10.1007 /$ s00198-019-05190-5

23. Neri SGR, Gadelha AB, David AC, Ferreira AP, Safons MP, Tiedemann A et al. The Association Between Body Adiposity Measures, Postural Balance, Fear of Falling, and Fall Risk in Older Community-Dwelling Women. J Geriatr Phys Ther. 2019;42(3):94100. doi: 10.1519/JPT.0000000000000165

24. Gadelha AB, Neri SGR, Vainshelboim B, Ferreira AP, Lima RM. Dynapenic abdominal obesity and the incidence of falls in older women: a prospective study. Aging Clin Exp Res. 2020;32(7):12631270. doi: $10.1007 / \mathrm{s} 40520-019-01318-z$

25. Pereira J, Elias JM, Neri SGR, Gadelha AB, Lemos RR, Lima RM. Dynapenic Abdominal Obesity as a Risk Factor for Falls in Older Women. Top Geriatr Rehabil. 2019;35(2):149-155. doi: 10.1097/ TGR.0000000000000225 